\title{
Immunosensoren - Universelles Protokoll zur Immobilisierung von Antikörpern für die spezifische Antigen-Detektion
}

\author{
Anja Henseleit ${ }^{1}$, Stefan Schmieder ${ }^{2}$, Elke Boschke ${ }^{1}$, Uwe Marx ${ }^{3,4}$, Silke Hofmann ${ }^{3}$, Reinhold A. \\ Klapsing ${ }^{5}$ und Frank Sonntag ${ }^{2}$ \\ ${ }^{1}$ TU Dresden Institut für Lebensmittel- und Bioverfahrenstechnik, 01069 Dresden, Bergstraße 120 \\ ${ }^{2}$ Fraunhofer-Institut für Werkstoff- und Strahltechnik IWS, 01277 Dresden, Winterbergstraße 28 \\ ${ }^{3}$ TU Berlin Institut für Biotechnologie, 13355 Berlin, Gustav-Meyer-Allee 25 \\ ${ }^{4}$ TissUse GmbH, 15528 Spreenhagen, Markgrafenstraße 18 \\ ${ }^{5}$ capitalis technology GmbH, 10117 Berlin, Krausenstraße 8
}

\begin{abstract}
Es wurde ein Protokoll zur stabilen und funktionellen Immobilisierung von Antikörpern über Protein A auf Goldoberflächen für den spezifischen Antigen-Nachweis entwickelt, charakterisiert, optimiert und erfolgreich etabliert. Die Verwendung von hydrophilen Polyethylenglykolketten als Blockungsreagenz wurde optimiert und in das Funktionalisierungsprotokoll integriert. Um mehrere sequentielle Bindungszyklen reproduzierbar auf einem Chip messen zu können, wurde Natriumhydroxid zur mehrmaligen Regeneration der Antikörper verwendet.

Mit zwei unterschiedlichen Antikörpern zur Detektion von phytopathogenen Viruspartikeln konnte die Eignung und die Universalität der erfolgreich adaptierten und etablierten Oberflächenchemie gezeigt werden. Es wurden Konzentrationsreihen vermessen. Die Nachweisgrenze für die phytopathogenen Viruspartikel lag bei $0,9 \mu \mathrm{g} / \mathrm{mL}$. Damit konnte ein Oberflächenplasmonenresonanz (SPR)-basierter Immunosensor zur Detektion von phytopathogenen Viruspartikeln entwickelt und höhere Sensitivitäten im Vergleich zu anderen, publizierten Funktionalisierungsprotokollen erreicht werden. Dank der Regeneration der Sensoroberfläche ließ sich die Effizienz der Messungen weiter steigern.
\end{abstract}

\section{Einleitung}

Immunosensoren sind Biosensoren, die auf der Spezifität von Liganden (Antikörpern) zu ihrem Analyt unter Ausbildung eines stabilen Immunkomplexes beruhen. Da sie durch den Einsatz von hochaffinen Antikörpern hohe Sensitivitäten aufweisen, ist ihr Einsatzgebiet vielfältig. So sind sie beispielsweise in der klinischen Diagnostik, der Umweltanalytik, der Militärtechnik, der Lebensmittelanalytik und der Prozesskontrolle vertreten.

Die SPR-Spektroskopie gehört zu den bevorzugt eingesetzten Detektionsmethoden, da sie Veränderungen des Brechungsindexes, hervorgerufen durch die Bildung des Immunkomplexes, ohne zusätzliche Modifizierung der Reaktionspartner erfassen kann. Dennoch existieren im Vergleich zur großen Anzahl an Veröffentlichungen nur wenige kommerzielle Anwendungen. Die Gründe dafür liegen in den noch nicht vollständig geklärten Vorgängen bei der Immobilisierung, der Orientierung und den spezifischen Eigenschaften der Antikörper auf der Sensoroberfläche. Durch die Immobilisierung und die damit einhergehende sterische Hinderung durch die Oberfläche sowie die zufällige Orientierung der Biomoleküle verringert sich häufig ein Teil der Bindungsaktivität der Antikörper. Um eine möglichst hohe Sensitivität des Immunosensors zu gewährleisten, ist es erforderlich, eine geeignete Immobilisierungsstrategie auszuwählen. Vor allem für Messungen in Realproben ist es entscheidend, dass die funktionalisierte Fläche eine ausreichende Immobilisierungsdichte und eine genügend hohe Bindungsaktivität besitzt, um auch geringe Analytkonzentrationen detektieren zu können.

Das Ziel des Projektes war es, ein Nachweissystem für intakte phytopathogene Viruspartikel in Echtzeit, ohne vorhergehende zeitaufwendige Aufbereitung, zu entwickeln. 
Dazu wurden ein polyklonaler und ein monoklonaler Antikörper ausgewählt, die spezifisch das Soil Borne Cereal Mosaic Virus (SBCMV) binden. Beide Antikörper sollten mittels einer geeigneten Methode möglichst funktionell und stabil auf die Goldfläche eines Sensorchips immobilisiert werden, um Konzentrationsreihen zu vermessen.

Das SBMCV wird über den Vektor des bodenbürtigen Polymyxa graminis in die verschiedenen Getreidesorten (Frischroggen, Tritikale und Weizen) übertragen und kann in den betroffenen Gebieten zu Ertragsverlusten von $50 \%$ bis $70 \%$ führen. Die einzige effektive Bekämpfungsmethode ist der Anbau von resistenten Sorten [1]. Diese Kulturpflanzen besitzen eine sogenannte Translokationsresistenz [2, 3]. Das bedeutet, dass der natürliche Virusbefall der Wurzeln zwar ungehindert geschieht, aber die Ausbreitung in die grünen Pflanzenteile nicht oder stark verzögert stattfindet [2, 3]. Für die Resistenzprüfung sind neben der visuellen Begutachtung vor allem sensitive, selektive, preiswerte sowie zeitnahe Nachweismethoden von entscheidender Bedeutung $[1,4]$.

\section{Material und Methoden}

\subsection{Geräte}

Alle beschriebenen SPR-Experimente wurden am Fraunhofer SPR-System durchgeführt [5, 6, 7]. Die Sensorchips (capitalis technology GmbH, Berlin, Deutschland) werden mittels Spritzguss aus TOPAS ${ }^{\circledR}$ als objektträgergroße Einwegartikel (76 x 26 x $4 \mathrm{~mm}^{3}$ ) gefertigt. In der Mitte des Chips befindet sich eine $50 \mathrm{~nm}$ dicke, $12 \times 3 \mathrm{~mm}^{2}$ große, rechteckige Goldschicht, welche den sensitiven Bereich bildet und auf der bis zu 180 Messflächen realisiert werden können [6]. Die Liganden werden mittels der Funktionalisierungsflusszelle (capitalis technology GmbH, Berlin, Deutschland) offline auf die Goldfläche immobilisiert.

\subsection{Materialien}

Zur Detektion der Viruspartikel wurden ein monoklonaler (SBCMV-mAk) und ein polyklonaler Antikörper (SBCMV-pAk) verwendet. Sowohl das SBCM Virus als auch beide Antikörper wurden vom Julius KühnInstitut (JKI) Bundesforschungsinstitut für Kulturpflanzen Quedlinburg Deutschland zur Verfügung gestellt. Protein A wurde von GE Healthcare Bio-Science AB Uppsala Schweden, DTSSP von Interchim Montluçon Frankreich und das thiolmodifizierte Polyethylenglycol (HO-PEG) von Iris Biotech GmbH Marktredwitz Deutschland bezogen. Als Puffer wurde PBST, zusammengesetzt aus 10 mM PBS pH 7,4 (Sigma-Aldrich Chemie GmbH, Steinheim, Deutschland) und 0,05\% (v/v) Tween 20 (Merck KGaA, Darmstadt, Deutschland) verwendet. Der Puffer wurde vor dem Gebrauch im Exsikkator entgast. Alle weiteren Chemikalien wurden von Merck KGaA Darmstadt Deutschland bezogen.

\subsection{Immobilisierungsprotokoll}

Zu Beginn eines SPR-Experiments wird die Goldfläche des Sensorchips wie folgt vorbereitet: (i) Reinigen der Goldfläche mit rauchender Salpetersäure (65\%). (ii) Inkubation des Sensorchips in Neutralisationslösung ( 1 x 25 \% Ammoniak, 1 x $30 \%$ Wasserstoffperoxid, 5 x ddH $\mathrm{d}_{2} \mathrm{O}$ ) für 2 min bei Raumtemperatur (RT). (iii) Spülen mit $\mathrm{ddH}_{2} \mathrm{O}$ und Trocknen mittels Stickstoffgas.

Für alle durchgeführten SPR-basierten Experimente wurde die 17-Kanal-Funktionalisierungsflusszelle zur Immobilisierung genutzt. Für die Detektion von SBCMV wurde folgendes Immobilisierungsprotokoll erarbeitet: (i) Inkubieren von $1 \mathrm{mg} / \mathrm{mL}$ Protein A für $1 \mathrm{~h}$ bei RT. (ii) Spülen der Fläche mit PBST. (iii) Inkubieren von $50 \mu \mathrm{g} / \mathrm{mL}$ Antikörper (SBCMV-pAk, SBCMV-mAk, Referenz-Ak) für $1 \mathrm{~h}$ bei RT. (iv) Spülen der Fläche mit PBST. (v) Blockung mit 1 mmol/L HO-PEG für $1 \mathrm{~h}$ bei RT. (vi) Spülen der Fläche mit PBST.

\subsection{SPR-Messungen}

Die SPR-Messungen wurden bei einer Fließrate von $2,48 \mu \mathrm{L} / \mathrm{s}$ als Doppelbestimmung wie folgend beschrieben durchgeführt: 
(i) Spülen der Fläche mit PBST. (ii) Injektion von $60 \mu \mathrm{L}$ SBCMV. (iii) Spülen der Fläche mit PBST. (iv) Regeneration mit $60 \mu \mathrm{L} 8 \mathrm{mmol} / \mathrm{L} \mathrm{NaOH}$. (v) Spülen der Fläche mit PBST.

10 mmol/L PBST wurde als Laufpuffer sowie zur Verdünnung der Proben verwendet.

\section{Ergebnisse und Diskussion}

Zur Abschätzung der Spezifität der detektierten Signale ist ein Referenzkanal unabdingbar [8]. Die Referenzfläche sollte möglichst ähnlich wie die Messfläche funktionalisiert sein, um alle wichtigen Effekte wie Temperatur-Schwankungen, mechanische Bewegungen und unspezifische Bindungen, die Brechungsindex-Änderungen verursachen können, zu erfassen und diese von den Bindungssignalen abziehen zu können [8,9]. Als Referenz wurde hier ein Antikörper (Referenz-Ak), der keine Affinität zu dem entsprechenden Antigen oder den Serumbestandteilen besitzt, auf die gleiche Weise wie der Detektionsantikörper immobilisiert.

Zur Ermittlung einer Nachweisgrenze und Erstellung der Kalibrierungskurven ${ }^{1}$ wurden Viruskonzentrationen von $0,7 \mu \mathrm{g} / \mathrm{mL}$ bis $92,5 \mu \mathrm{g} / \mathrm{mL}$ - enspricht in etwa den gefundenen Detektionsgrenzen - online auf zuvor nach dem erarbeiteten Immobilisierungsprotokoll funktionalisierten Chips injiziert. Da während des Regenerationsscoutings ermittelt wurde, dass $60 \mu \mathrm{L} 8 \mathrm{mmol} / \mathrm{L} \mathrm{NaOH}$ maximal viermal injiziert werden kann, ohne größere Abweichungen zu riskieren, wurde die Konzentrationsreihe geteilt und auf mehreren Sensorchips vermessen. Die Sensorgramme ausgewählter Konzentrationen sind in Bild 1 dargestellt.

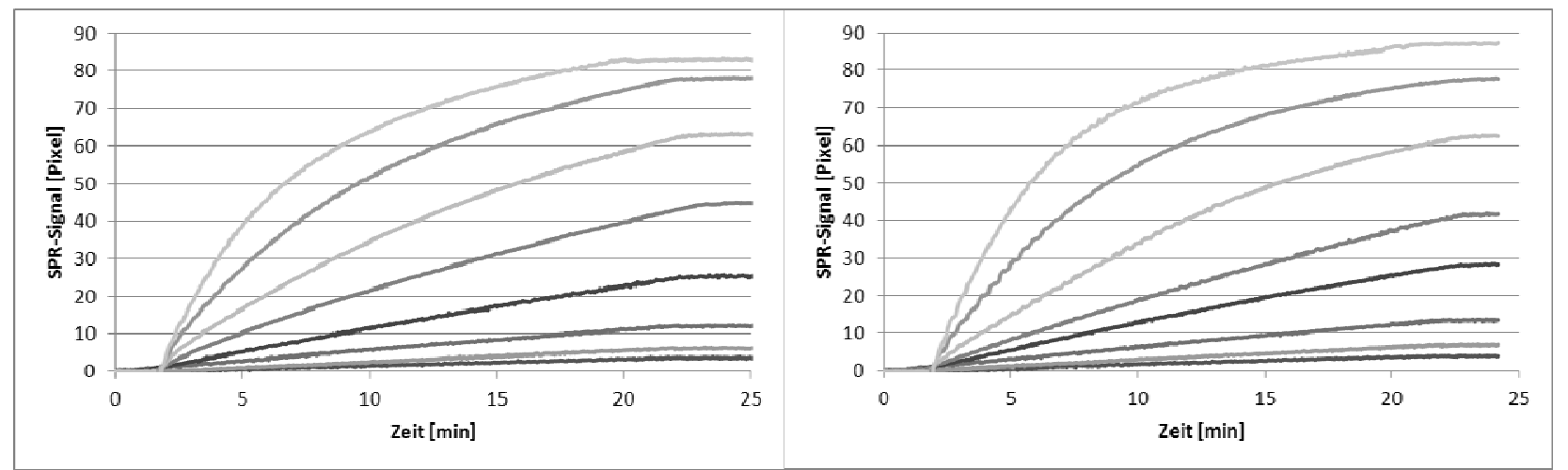

Bild 1 Sensorgramm der SBCMV-Konzentrationsreihe (von unten nach oben: 0,7 $\mu \mathrm{g} / \mathrm{mL} ; 1,4 \mu \mathrm{g} / \mathrm{mL} ; 2,9 \mu \mathrm{g} / \mathrm{mL}$; 5,8 $\mu \mathrm{g} / \mathrm{mL} ; 11,6 \mu \mathrm{g} / \mathrm{mL} ; 23,1 \mu \mathrm{g} / \mathrm{mL} ; 46,2 \mu \mathrm{g} / \mathrm{mL}$ und 92,5 $\mu \mathrm{g} / \mathrm{mL}$ ) auf SBCMV-pAk (links) und auf SBCMV-mAk (rechts) funktionalisierten Flächen. Die Signale des Referenz-Ak wurden von den Bindungssignalen abgezogen.

Das Detektionslimit des Fraunhofer SPR-Systems liegt bei etwa drei Pixeln. Folglich ist die SBCMVKonzentration von $0,9 \mu \mathrm{g} / \mathrm{mL}$ die Nachweisgrenze.

Die minimal detektierbare Konzentration hängt von zahlreichen Faktoren wie unspezifischen Bindungen, Massentransport, der Sensitivität des Sensors sowie von der Antikörper-Antigen Bindungseffektivität ab [9].

Da die Aktivität der Antikörper und damit die Antikörper-Antigen Bindungseffektivität stark durch die angewandte Immobilisierungsmethode beeinflusst wird, sind die Sensitivitäten von unterschiedlichen Sensoren nur schwer vergleichbar, wenn nicht dieselben Immobilisierungsmethoden und Antikörper benutzt wurden [10]. So ermittelten Torrance und Mitarbeiter für die Detektion des phytopathogenen Cowpea Mosaic Virus mittels Biacore X eine Nachweisgrenze von 12,5 $\mu \mathrm{g} / \mathrm{mL}$ [11]. Sie fixierten die gegen den Virus gerichteten, Cystein-modifizierten variablen Antikörperfragmente mittels N-Maleoyl-SuccinimidylEster an die Goldfläche des Sensorchips. Kim und Mitarbeiter immobilisierten mAk gegen den Oyster Mushroom Spherical Virus mittels NHS/EDC (N-Hydroxysuccinimid / 1-Ethyl-3-(3-Di-methylaminopropyl)Carbodiimid) auf die carboxymethylierte Dextranschicht der Sensorchips des Autolab Esprit SPRSystems [12]. Damit konnten sie Viruspartikel bis zu einer Konzentration von 1,36 $\mu \mathrm{g} / \mathrm{mL}$ detektieren.

\footnotetext{
1 Aus den Bindungssignalen der Viruskonzentrationen können logarithmische Kalibrierungskurven erstellt und Realproben mit unbekannter Viruskonzentration vermessen werden.
} 


\section{Zusammenfassung und Ausblick}

Ein Protokoll zur stabilen und funktionellen Immobilisierung von Antikörpern über Protein A auf Goldoberflächen wurde entwickelt, optimiert und erfolgreich etabliert und die Anwendung von Polyethylenglykol zum Blocken gegen unspezifische Bindungen in das Funktionalisierungsprotokoll integriert.

Mit einer pH-Wertänderung konnten die Antikörper mehrmals regeneriert und so mehrere sequentielle Bindungszyklen reproduzierbar auf einem Chip gemessen werden.

Da das entwickelte Funktionalisierungsprotokoll die Regeneration der Sensoroberfläche erlaubt, konnte eine weitere Effizienzsteigerung erzielt werden.

Für die Detektion der Viruspartikel wurden Konzentrationsreihen vermessen. Die ermittelte Nachweisgrenze liegt bei ca. 0,9 $\mu \mathrm{g} / \mathrm{mL}$. Damit konnte ein Immunosensor zur Detektion von phytopathogenen Viruspartikeln entwickelt werden, der höhere Sensitivitäten im Vergleich zu anderen Funktionalisierungsprotokollen aufweist $[11,12]$.

In weiterführenden Projekten soll anhand der zellbasierten Substanzcharakterisierung an humanen Leberzellen die Eignung und die Universalität der hier verwendeten Oberflächenchemie demonstriert werden. Dafür wird als Marker unter anderem humanes Serumalbumin genutzt, da dieses das von der Leber am stärksten synthetisierte Protein darstellt.

\section{Literatur}

[1] K. Kanyuka, D. J. Lovell, O. P. Mitrofanova, K. Hammond-Kosack, M. J. Adams: Plant Pathology, 53(2), 154-160: A controlled environment test for resistance to Soil-borne cereal mosaic virus (SBCMV) and its use to determine the mode of inheritance of resistance in wheat cv. Cadenza and for screening Triticum monococcum genotypes for sources of SBCMV resistance. 2004.

[2] T. Kühne: Virus Research, 141(2), 174-183: Soil-borne viruses affecting cereals--Known for long but still a threat. 2009.

[3] F. Ordon, A. Habekuss, U. Kastirr, F. Rabenstein, T. Kühne: Journal of Phytopathology, 157(9), 535-545: Virus Resistance in Cereals: Sources of Resistance, Genetics and Breeding. 2009.

[4] C. Vaïanopoulos, A. Legrève, V. Moreau, C. Bragard: Journal of Virological Methods, 159(2), 227-232: Broad-spectrum detection and quantitation methods of Soil-borne cereal mosaic virus isolates. 2009.

[5] B. Barlen, S. Datta Mazumdar, N. Danz, F. Sonntag, M. Keusgen: BIOS: A new handheld surfaceplasmon-resonance device for rapid on-location detection of bacterial infections. 2008.

[6] M. Mertig, A. Kick, M. Bonsch, B. Katzschner, J. Voigt, F. Sonntag, N. Schilling, U. Klotzbach, N. Danz, S. Begemann, A. Herr, M. Jung: Sensors 2009 IEEE, 392 - 395: A novel platform technology for the detection of genetic variations by surface plasmon resonance. 2009.

[7] F. Sonntag, S. Schmieder, A. Lubitz, C. Giese, U. Marx, N. Danz, U. Klotzbach: Dresdner Beiträge Medizintechnik: SPR basierte Parameterbestimmung für immunologische in vitro Testung von Substanzen mit künstlichen humanen Lymphknoten. 2008.

[8] B. H. Schneider, R. L. Dickinson, M. D. Vach, J. V. Hoijer, L. V. Howard: Biosensors \& Bioelectronics, 15(1-2), 13-22: Highly sensitive optical chip immunoassays in human serum. 2000.

[9] J. Xu, D. Suarez, D. S. Gottfried: Analytical and Bioanalytical Chemistry, 389(4), 1193-1199: Detection of avian influenza virus using an interferometric biosensor. 2007.

[10] P. D. Skottrup, M. Nicolaisen, A. F. Justesen: Biosensors and Bioelectronics, 24(3), 339-348: Towards on-site pathogen detection using antibody-based sensors. 2008.

[11] L. Torrance, A. Ziegler, H. Pittman, M. Paterson, R. Toth, I. Eggleston: Journal of Virological Methods, 134(1-2), 164-170: Oriented immobilisation of engineered single-chain antibodies to develop biosensors for virus detection. 2006.

[12] S. Kim, M. Kim, J. Kim, H. Lee, H. Ro: Journal of Virological Methods, 148(1-2), 120-124: Detection of the mycovirus OMSV in the edible mushroom, Pleurotus ostreatus, using an SPR biosensor chip. 2008. 